\title{
Dynamics of a New Hyperchaotic System with Only One Equilibrium Point
}

\author{
Xiang Li and Ranchao Wu \\ School of Mathematics, Anhui University, Hefei 230039, China \\ Correspondence should be addressed to Ranchao Wu; rcwu@ahu.edu.cn \\ Received 24 April 2013; Revised 27 June 2013; Accepted 2 July 2013 \\ Academic Editor: NanJing Huang
}

Copyright ( $92013 \mathrm{X}$. Li and R. Wu. This is an open access article distributed under the Creative Commons Attribution License, which permits unrestricted use, distribution, and reproduction in any medium, provided the original work is properly cited.

A new 4D hyperchaotic system is constructed based on the Lorenz system. The compound structure and forming mechanism of the new hyperchaotic attractor are studied via a controlled system with constant controllers. Furthermore, it is found that the Hopf bifurcation occurs in this hyperchaotic system when the bifurcation parameter exceeds a critical value. The direction of the Hopf bifurcation as well as the stability of bifurcating periodic solutions is presented in detail by virtue of the normal form theory. Numerical simulations are given to illustrate and verify the results.

\section{Introduction}

Since Lorenz found the first chaotic attractor in the 3D autonomous chaotic system in 1963 [1], people have realized that chaos is a ubiquitous and extremely complex nonlinear phenomenon in nature. In the past few years, motivated by many unknown interesting properties and some potential practical applications, great efforts have been made in constructing chaotic and hyperchaotic systems. Particularly, Chen and Ueta found a new chaotic system, called the Chen system [2] in 1999, based on Lorenz system. Afterwards, Lü and Chen furthermore found a chaotic system [3], which represents the transition between the Lorenz system and the Chen system. Some other new chaotic systems, including the Liu system $[4,5]$ and T system $[6]$, have also been constructed and investigated in recent years.

Recently, applications of hyperchaos have become a central topic in research. Some interesting hyperchaotic systems were presented in the past two decades, and their dynamics have been investigated extensively owing to their useful potential applications in engineering. Historically, hyperchaos was firstly reported by Rössler in 1979 [7], which was the noted 4D hyperchaotic Rössler system. A hyperchaotic system is usually defined as a chaotic system with more than one positive Lyapunov exponent. It means that hyperchaotic systems have more complex dynamical behaviors than chaotic systems [8-14]. These complex dynamics could be explored via bifurcation analysis of systems with varying parameters. Nowadays, bifurcation is one of most active research topics in the field of nonlinear science [15-17].

Now in this paper, based on the Lorenz system, a new four-dimensional hyperchaotic system with only one equilibrium point is constructed. Some basic dynamical properties, such as the Lyapunov exponents, bifurcation diagram, fractal dimensions, and hyperchaotic behaviors of this new system are investigated. Furthermore, the compound structure and forming mechanism of the new hyperchaotic attractor are studied by a controlled system with constant controllers. It is found that the two single scroll attractors, which form the complete compound hyperchaotic attractor, merely originate from some simple limit circles. As is well known, the Hopf bifurcations can give rise to limit circles. Therefore, the Hopf bifurcation analysis is carried out to investigate its complex dynamical behaviors. See that a Hopf bifurcation occurs in this system when the bifurcation parameter exceeds a critical value. The direction of the Hopf bifurcation and the stability of bifurcating periodic solutions are also presented by applying the normal form theory. 


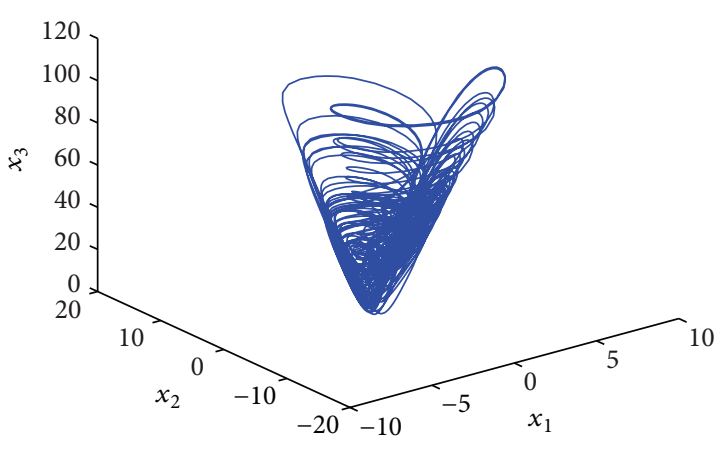

FIGURE 1: Hyperchaotic attractor of system (2).

\section{A New 4D Hyperchaotic System}

In [1], the Lorenz system is given by

$$
\begin{gathered}
\dot{x}=a(y-x), \\
\dot{y}=c x-x z-y, \\
\dot{z}=x y-b z,
\end{gathered}
$$

which is chaotic when $a=10, b=8 / 3$, and $c=28$.

Based on the Lorenz system, a new four-dimensional system is expressed as

$$
\begin{gathered}
\dot{x}_{1}=a\left(x_{2}-x_{1}\right), \\
\dot{x}_{2}=c x_{1}-x_{1} x_{3}-x_{2}+e x_{4}, \\
\dot{x}_{3}=x_{1}^{4}+x_{2}^{2}-b x_{3}, \\
\dot{x}_{4}=-d x_{2},
\end{gathered}
$$

where $x_{1}, x_{2}, x_{3}$, and $x_{4}$ are state variables and $a, b, c, d$, and $e$ are parameters.

Here, let

$$
a>0, \quad b>0, \quad d e>0 .
$$

By simple computation, it is easy to obtain that system (2) has only one equilibrium point $O(0,0,0,0)$ and the Jacobian matrix $J$ at the equilibrium point $O(0,0,0,0)$ is

$$
J=\left(\begin{array}{cccc}
-a & a & 0 & 0 \\
c & -1 & 0 & e \\
0 & 0 & -b & 0 \\
0 & -d & 0 & 0
\end{array}\right) .
$$

Thus the corresponding characteristic equation can be obtained as

$$
\begin{aligned}
& f(\lambda) \\
& =(\lambda+b) \\
& \quad \times\left[\lambda^{3}+(a+1) \lambda^{2}+(a+d e-a c) \lambda+a d e\right]=0 .
\end{aligned}
$$

According to the Routh-Hurwitz criterion, the real parts of the roots for (5) are all negative if and only if parameters satisfy the condition

$$
c<1+\frac{d e}{\left(a^{2}+a\right)} .
$$

Thus, one can get the following.

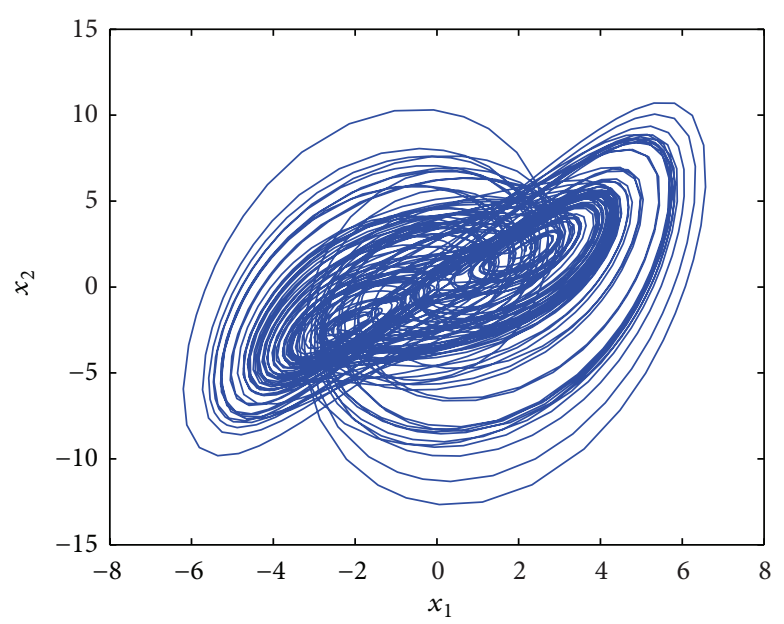

FIGURE 2: Hyperchaotic attractor in $x_{1}-x_{2}$ plane.

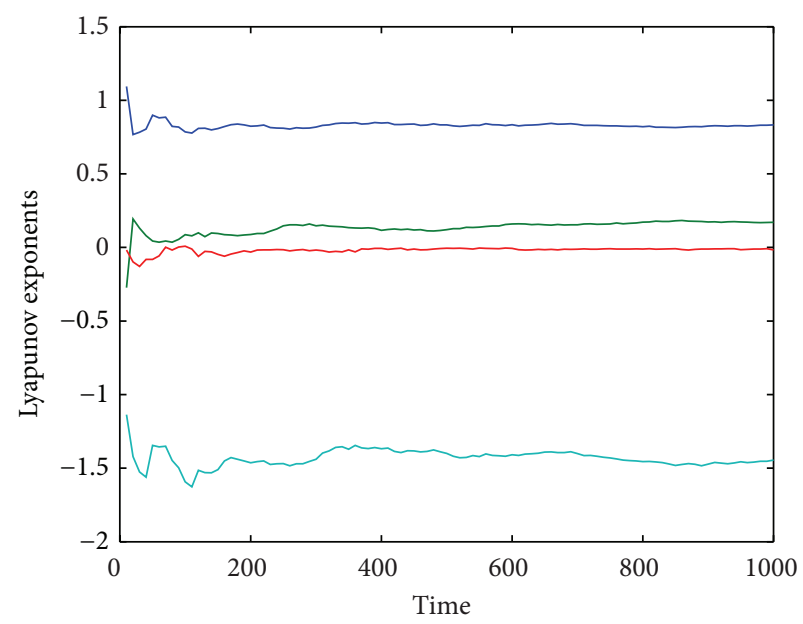

Figure 3: The Lyapunov exponent spectrum.

Theorem 1. For the only one equilibrium point $\mathrm{O}(0,0,0,0)$ of system (2),

(1) when $c<1+d e /\left(a^{2}+a\right)$, the equilibrium $O$ is asymptotically stable;

(2) when $c \geq 1+d e /\left(a^{2}+a\right)$, the equilibrium $O$ is unstable.

When $a=10, b=8 / 3, c=28, d=2, e=12$, using MATLAB software, it is easy to show that the eigenvalues are $\lambda_{1}=-22.4395, \lambda_{2}=10.4123, \lambda_{3}=1.0272$, and $\lambda_{4}=$ -2.6667 . Hence, the equilibrium $O$ is an unstable saddle, and the four Lyapunov exponents are, respectively, to be

$$
\begin{gathered}
\lambda_{1}=0.8331, \quad \lambda_{2}=0.1703, \\
\lambda_{3}=0, \quad \lambda_{4}=-1.4456 .
\end{gathered}
$$

Obviously, there are two positive Lyapunov exponents. Therefore, the new $4 \mathrm{D}$ system (2) is a hyperchaotic system, and the hyperchaotic attractor is shown in Figures 1 and 2. The Lyapunov exponent spectrum is shown in Figure 3, and the bifurcation diagram of state variable $x_{2}$ with parameter $c$ is shown in Figure 4. 


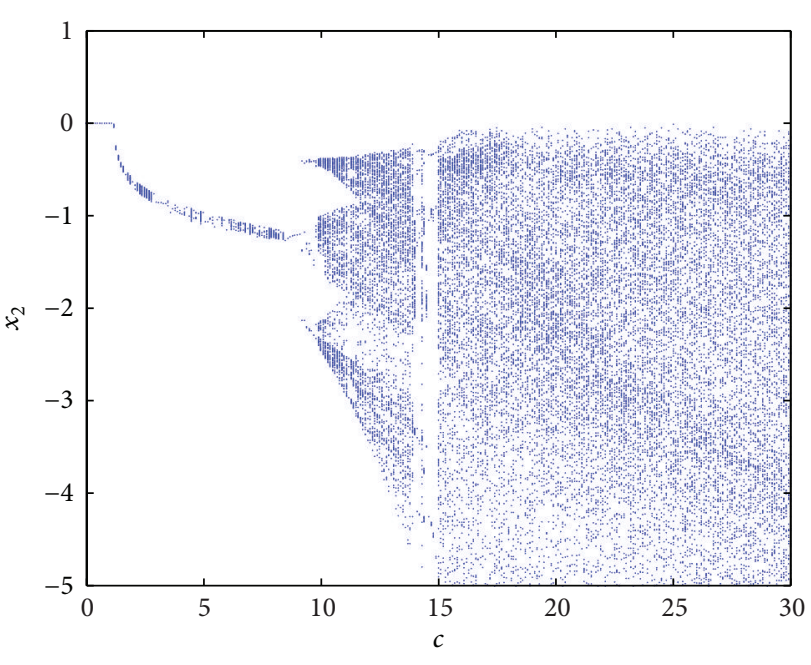

FIgURE 4: Bifurcation diagram of system (2) versus $c$.

\section{Analysis of Basic Dynamic Behaviors}

3.1. Symmetry. The new hyperchaotic system (2) is invariant under the coordinate transformation $\left(x_{1}, x_{2}, x_{3}, x_{4}\right) \rightarrow$ $\left(-x_{1},-x_{2}, x_{3},-x_{4}\right)$. That is, under reflection about the $z$-axis, the symmetry persists for all values of the system parameters.

3.2. Dissipation and the Existence of an Attractor. The divergence of system (2) is defined by

$$
\begin{aligned}
\nabla V & =\frac{\partial \dot{x}_{1}}{\partial x_{1}}+\frac{\partial \dot{x}_{2}}{\partial x_{2}}+\frac{\partial \dot{x}_{3}}{\partial x_{3}}+\frac{\partial \dot{x}_{4}}{\partial x_{4}} \\
& =-a-1-b .
\end{aligned}
$$

When $a=10, b=8 / 3, \nabla V=-41 / 3<0$, so system $(2)$ is a dissipative system and converges with an index rate of $e^{-(41 / 3) t}$. Volume element $V_{0}$ shrinks to $V_{0} e^{-(41 / 3) t}$ at the time $t$. When $t \rightarrow \infty$, volume element $V_{0}$ shrinks to 0 . Therefore, all trajectories of the system will be confined to a congregation, whose volume is 0 . And the gradual movement behaviors are fixed into an attractor.

3.3. The Lyapunov Dimension. As we know, the Lyapunov exponents measure the exponential rates of divergence or convergence of nearby trajectories in phase space. The four Lyapunov exponents of system (2) are, respectively, $\lambda_{1}=$ $0.8331, \lambda_{2}=0.1703, \lambda_{3}=0, \lambda_{4}=-1.4456$, and when $a=10, b=8 / 3, c=28, d=2$, and $e=12$. The Lyapunov dimension of chaotic attractor of this new hyperchaotic system is fractional, which is described as

$$
\begin{aligned}
D_{L} & =j+\frac{1}{\left|\lambda_{j+1}\right|} \sum_{i=1}^{j} \lambda_{i} \\
& =3+\frac{1}{|-1.4456|}(0.8331+0.1703+0) \\
& =3.6941 .
\end{aligned}
$$

\section{Complexity and Forming Mechanism of the Attractor}

In order to investigate the complex structure and forming mechanism of the new hyperchaotic attractor, its controlled system is proposed and expressed as

$$
\begin{gathered}
\dot{x}_{1}=a\left(x_{2}-x_{1}\right), \\
\dot{x}_{2}=c x_{1}-x_{1} x_{3}-x_{2}+e x_{4}, \\
\dot{x}_{3}=x_{1}^{4}+x_{2}^{2}-b x_{3}, \\
\dot{x}_{4}=-d x_{2}+u,
\end{gathered}
$$

where $u$ is a constant controller, which can effectively control the dynamical behavior of the new hyperchaotic system.

It is found that the new hyperchaotic attractor of system (2) is evolved into the left half attractor when $u=-2.9$, while one can get the right half attractor when $u=2.9$, which is the mirror operation of the left half attractor. It means that the new hyperchaotic attractor of system (2) has a compound structure. That is, it can be obtained by merging together two single scroll attractors (left half attractor and right half attractor) which are shown in Figures 5 and 6, respectively, after performing one mirror operation.

Next, the forming mechanism of the new hyperchaotic attractor will be revealed by changing the value of the constant controller $u$ within a certain range. Here, the parameters of the controlled system (10) are chosen as $a=10, b=8 / 3$, $c=28, d=2$, and $e=12$, and its initial values are selected as $(0,1,0,0)$.

(1) When $|u| \geq 9.4$, the hyperchaotic attractor evolves into the convergent trajectories, which are shown in Figure 7.

(2) When $3.14<|u| \leq 9.3$, the hyperchaotic attractor evolves into the periodic trajectories, which are shown in Figure 8.

(3) When $2.9<|u| \leq 3.14$, the hyperchaotic attractor evolves into the period-doubling bifurcations, which are shown in Figure 9.

(4) When $2.8<|u| \leq 2.9$, the hyperchaotic attractor evolves into left half attractor (right half attractor), which is shown in Figures 5 and 6, respectively.

(5) When $0.5<|u| \leq 2.8$, the hyperchaotic attractor evolves into partial one but is still bounded in time, which is shown in Figure 10.

(6) When $|u| \leq 0.5$, the complete hyperchaotic attractor is obtained and shown in Figure 11.

The bifurcation diagram of state variable $x_{2}$ versus constant controller $u$ is shown in Figure 12. It is easy to see that the hyperchaotic attractor disappears when $|u|$ is large enough; when $|u|$ is small enough, a complete hyperchaotic attractor appears. Meanwhile, it has been found that its compound structure can be obtained by merging together two single scroll attractors after performing one mirror operation. However, the two single scroll attractors merely originate from some simple limit circles as shown in Figure 8 and are obtained after the period-doubling bifurcations. 


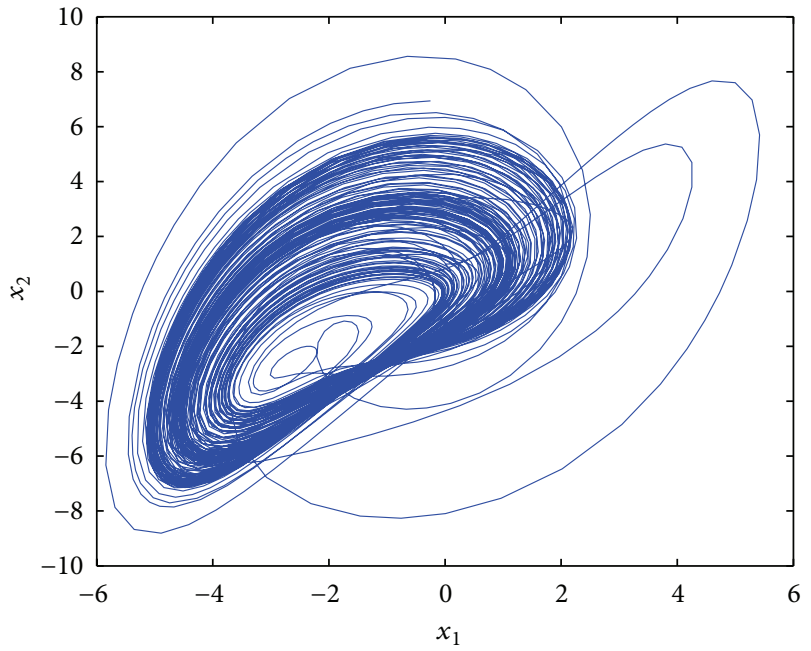

FIGURE 5: Left half attractor $(u=-2.9)$.

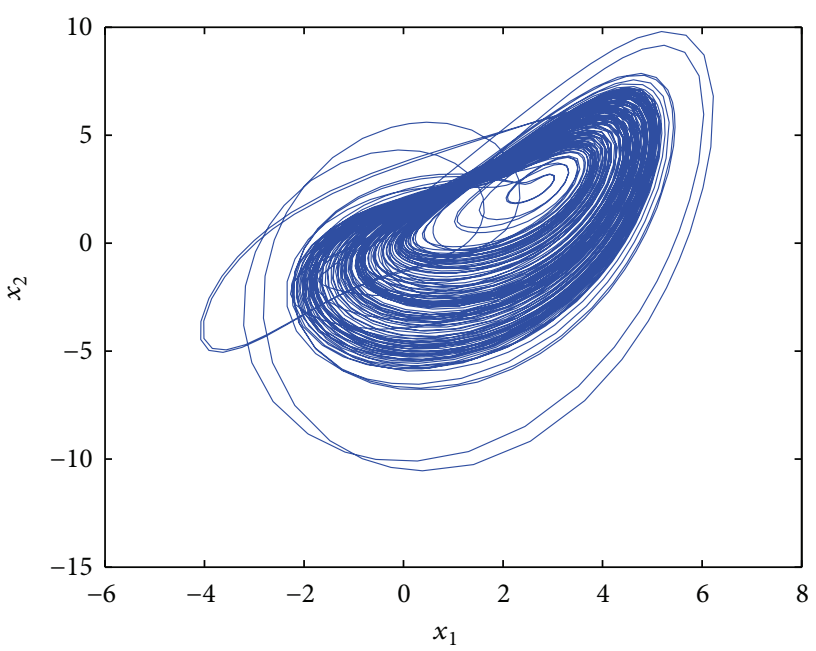

FIgURE 6: Right half attractor $(u=2.9)$.

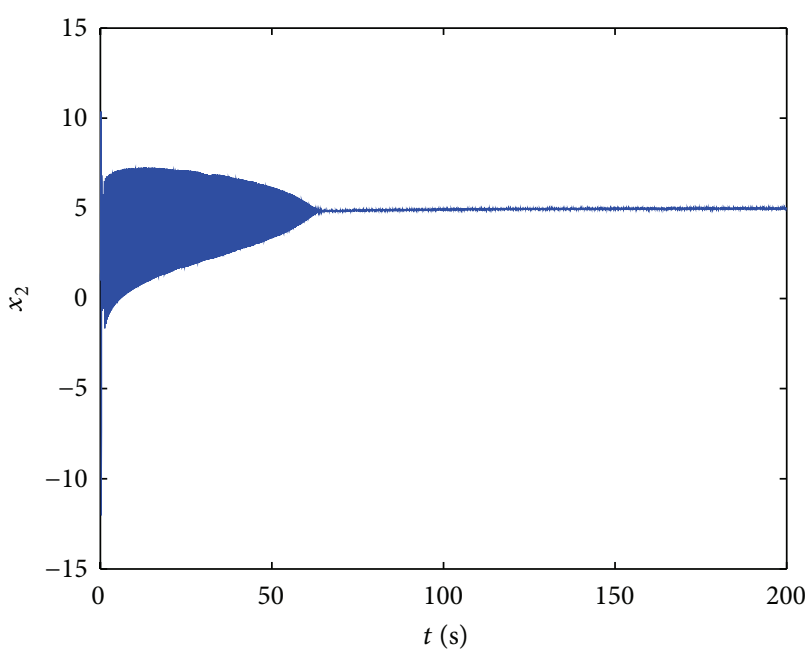

FiguRE 7: State trajectory of $x_{2}(u=10)$.

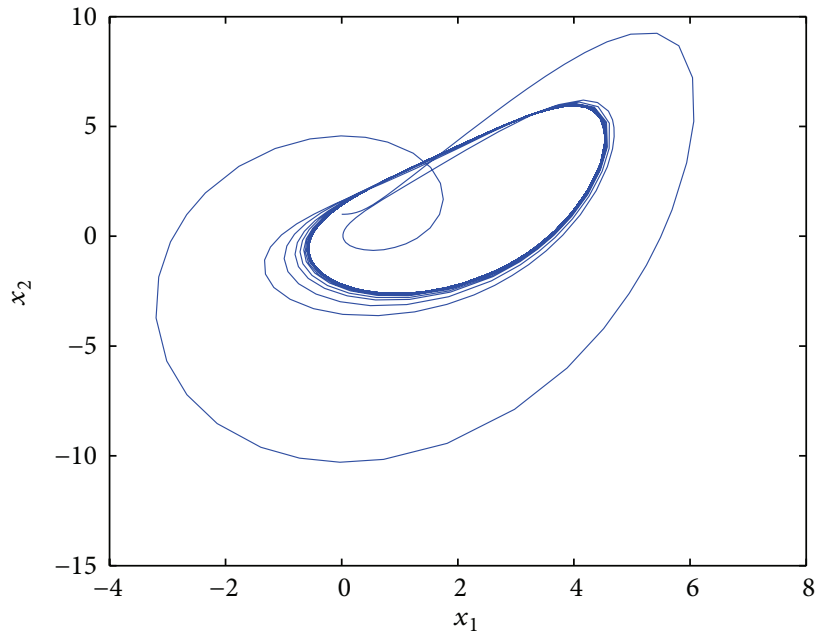

Figure 8: Periodic trajectories in $x_{1}-x_{2}$ plane $(u=3.5)$.

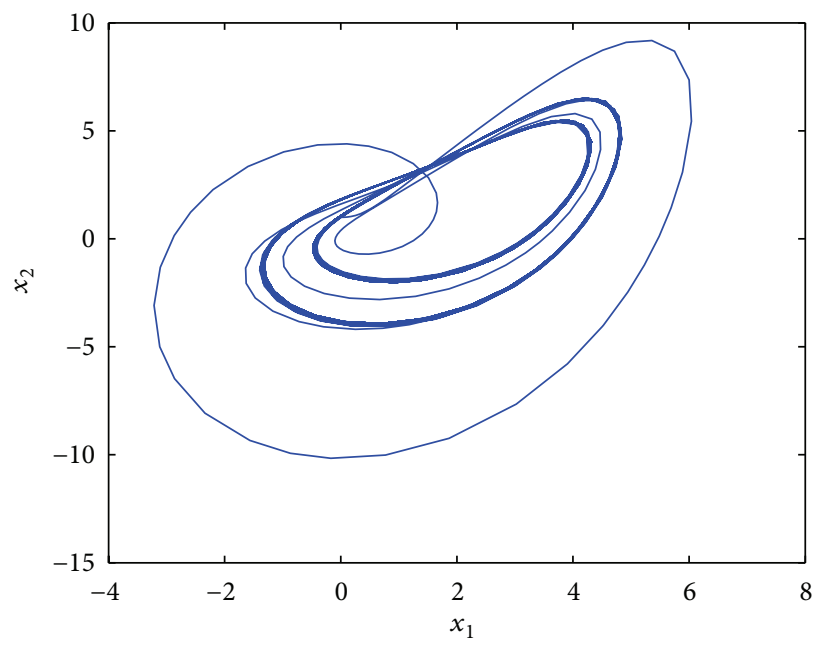

Figure 9: Period-doubling bifurcation in $x_{1}-x_{2}$ plane $(u=3.1)$.

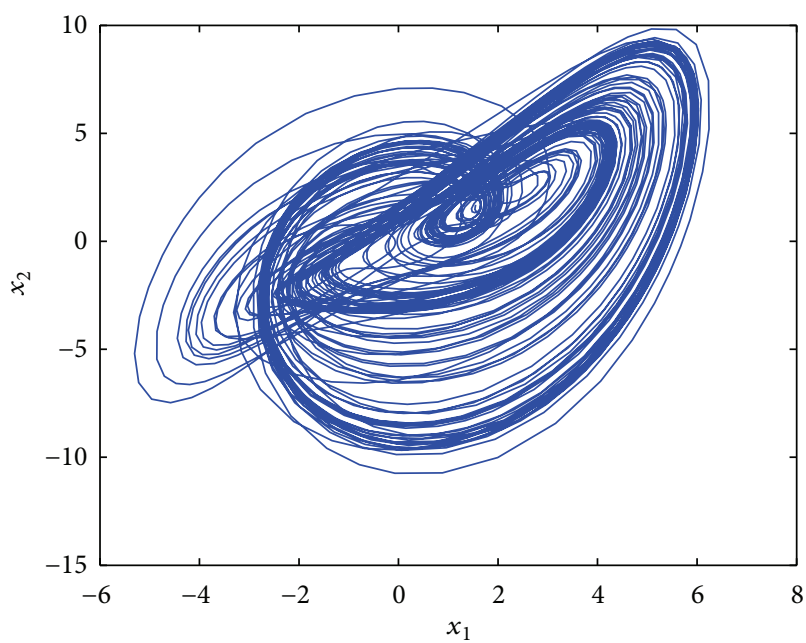

FIGURE 10: Hyperchaotic attractor in $x_{1}-x_{2}$ plane $(u=1.5)$. 


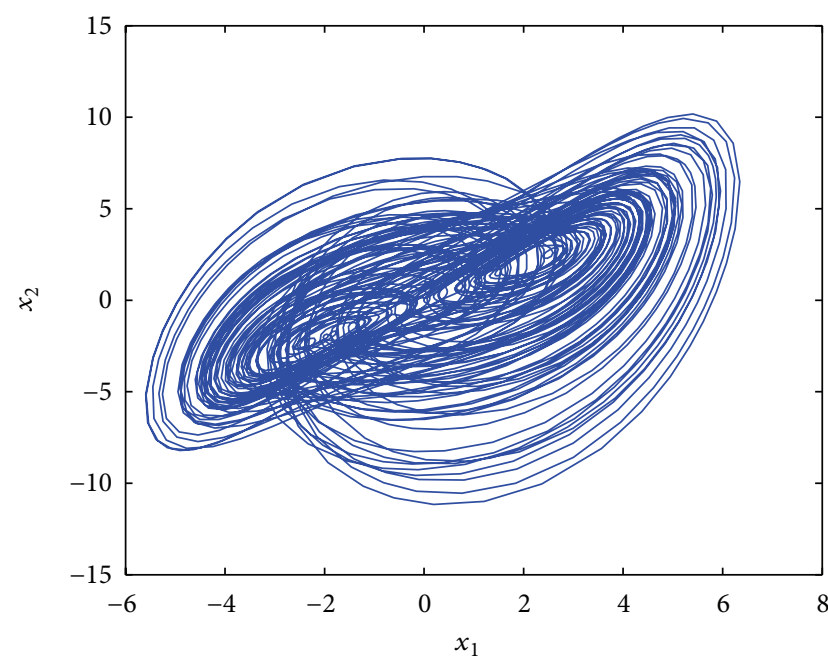

FIGURE 11: Complete hyperchaotic attractor in $x_{1}-x_{2}$ plane $(u=$ $0.2)$.

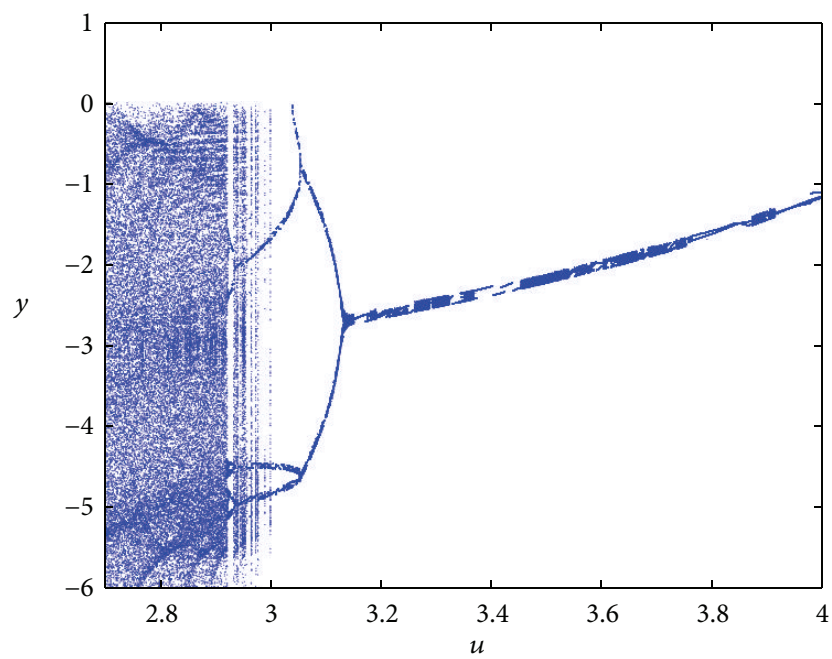

FIGURE 12: Bifurcation diagram of system (2) versus $u$.

\section{Hopf Bifurcation in the New Hyperchaotic System}

In Section 4, the compound structure and forming mechanism of the new hyperchaotic attractor are studied via detailed numerical simulations as well as theoretical analysis. It is shown that some simple limit circles are very important for evolution of single scroll chaotic attractors. Since the Hopf bifurcations can give rise to limit circles, then the Hopf bifurcation in the new hyperchaotic system (2) will be further discussed in this section.

5.1. Existence of Hopf Bifurcation. The equilibrium point $\mathrm{O}(0,0,0,0)$ is asymptotically stable when inequality $c<1+$ $d e /\left(a^{2}+a\right)$ holds. The critical value for the stability can be derived to be $c_{0}=1+d e /\left(a^{2}+a\right)$. Let $c=c_{0}$. Equation (5) can be rewritten into

$$
(\lambda+b)(\lambda+a+1)\left(\lambda^{2}+\frac{a d e}{a+1}\right)=0 .
$$

Obviously, (11) has four roots as follows, with a pair being purely imaginary conjugate:

$$
\begin{gathered}
\lambda_{1}=i \sqrt{\frac{a d e}{a+1}}=i \omega_{0}, \quad \lambda_{2}=-i \sqrt{\frac{a d e}{a+1}}=-i \omega_{0}, \\
\lambda_{3}=-(a+1), \quad \lambda_{4}=-b .
\end{gathered}
$$

Then, according to (5), one has

$$
\begin{aligned}
\lambda^{\prime}(c)= & (a \lambda(\lambda+b \lambda)) \\
\times( & 4 \lambda^{3}+(a+b+1) \lambda^{2} \\
& +2(a+d e-a c+a b+b) \lambda \\
& +a b(1-c)+d e(a+b))^{-1} .
\end{aligned}
$$

Therefore,

$$
\begin{aligned}
& \operatorname{Re}(\left.\lambda^{\prime}\left(c_{0}\right)\right) \\
&=\left(a b^{2}(a+1)^{3}+a^{2} d e(a+1)^{2}\right) \\
& \times\left(2 \left[b^{2}(a+1)^{4}+a d e(a+1)^{3}\right.\right. \\
&\left.\left.+a b d e(a+1)^{2}+a b^{2} d e(a+1)+a^{2} d^{2} e^{2}\right]\right)^{-1}, \\
& \operatorname{Im}\left(\lambda^{\prime}\left(c_{0}\right)\right) \quad\left(\left[a b^{2}(a+1)^{2}+a^{2} d e(a+1)\right] \omega_{0}\right) \\
& \quad \times\left(2 \left[b^{2}(a+1)^{4}+a d e(a+1)^{3}\right.\right. \\
& \quad+a b d e(a+1)^{2} \\
&\left.\left.\quad+a b^{2} d e(a+1)+a^{2} d^{2} e^{2}\right]\right)^{-1} .
\end{aligned}
$$

Obviously, $\operatorname{Re}\left(\lambda^{\prime}\left(c_{0}\right)\right) \neq 0$, so the Hopf bifurcation theorem [18] holds. Hence, when $c=c_{0}=1+d e /\left(a^{2}+a\right)$, system (2) undergoes a Hopf bifurcation at the equilibrium point $O(0,0,0,0)$. The above analysis is summarized as follows.

Theorem 2 (existence of the Hopf bifurcation). When $c$ passes through the critical value $c_{0}=1+d e /\left(a^{2}+a\right)$, system (2) undergoes a Hopf bifurcation at the equilibrium point.

5.2. Direction and Stability of Bifurcating Periodic Solutions. The direction, stability, and period of bifurcating periodic solutions for system (2) will be investigated in detail by virtue 
of the normal form theory [18]. The eigenvectors $v_{1}, v_{3}, v_{4}$ associated with $\lambda_{1}=i \omega_{0}, \lambda_{3}=-(a+1), \lambda_{4}=-b$ are, respectively,

$$
\begin{gathered}
v_{1}=\left(\begin{array}{c}
\frac{-a d e+i a \sqrt{a d e(a+1)}}{a\left(a^{2}+a+d e\right)} \\
\frac{i \sqrt{a d e(a+1)}}{d(a+1)} \\
0 \\
1
\end{array}\right), \\
v_{3}=\left(\begin{array}{c}
\frac{-a(a+1)}{d} \\
\frac{a+1}{d} \\
0 \\
1 \\
0 \\
0 \\
1 \\
0
\end{array}\right) .
\end{gathered}
$$

Define

$P$

$$
\begin{aligned}
& =\left(\operatorname{Re} v_{1}, \operatorname{Im} v_{1}, v_{3}, v_{4}\right) \\
& =\left(\begin{array}{cccc}
\frac{-a e}{\left(a^{2}+a+d e\right)} & \frac{a \sqrt{a d e(a+1)}}{d\left(a^{2}+a+d e\right)} & \frac{-a(a+1)}{d} & 0 \\
0 & \frac{\sqrt{a d e(a+1)}}{d(a+1)} & \frac{a+1}{d} & 0 \\
0 & 0 & 0 & 1 \\
1 & 0 & 1 & 0
\end{array}\right) \text {, } \\
& \left(x_{1}, x_{2}, x_{3}, x_{4}\right)^{T}=P\left(y_{1}, y_{2}, y_{3}, y_{4}\right)^{T} \text {. } \\
& \dot{y}_{1}=-\omega_{0} y_{2}+F_{1}\left(y_{1}, y_{2}, y_{3}, y_{4}\right), \\
& \dot{y}_{2}=\omega_{0} y_{1}+F_{2}\left(y_{1}, y_{2}, y_{3}, y_{4}\right) \text {, } \\
& \dot{y}_{3}=-(a+1) y_{3}+F_{3}\left(y_{1}, y_{2}, y_{3}, y_{4}\right) \text {, } \\
& \dot{y}_{4}=-b y_{4}+F_{4}\left(y_{1}, y_{2}, y_{3}, y_{4}\right) \text {, }
\end{aligned}
$$

where

$$
\begin{gathered}
F_{1}\left(y_{1}, y_{2}, y_{3}, y_{4}\right)=-r y_{4}\left(l y_{1}+m y_{2}+n y_{3}\right)+e r\left(y_{1}+y_{3}\right), \\
F_{2}\left(y_{1}, y_{2}, y_{3}, y_{4}\right)=-s y_{4}\left(l y_{1}+m y_{2}+n y_{3}\right)+\operatorname{er}\left(y_{1}+y_{3}\right), \\
F_{3}\left(y_{1}, y_{2}, y_{3}, y_{4}\right)=-F_{1}\left(y_{1}, y_{2}, y_{3}, y_{4}\right), \\
F_{4}\left(y_{1}, y_{2}, y_{3}, y_{4}\right)=\left(l y_{1}+m y_{2}+n y_{3}\right)^{4}+\left(p y_{2}+q y_{3}\right)^{2},
\end{gathered}
$$

in which

$$
\begin{gathered}
l=\frac{-a e}{a^{2}+a+d e}, \\
m=\frac{a \sqrt{a d e(a+1)}}{d\left(a^{2}+a+d e\right)}, \quad n=\frac{-a(a+1)}{d}, \\
p=\frac{\sqrt{a d e(a+1)}}{d(a+1)}, \quad r=\frac{-d(a+1)}{a^{3}+3 a^{2}+3 a+a d e+1}, \\
q=\frac{a+1}{d}, \\
s=\frac{a d(a+1)\left(a^{2}+2 a+d e+1\right)}{\sqrt{a d e(a+1)}\left(a^{3}+3 a^{2}+3 a+a d e+1\right)} .
\end{gathered}
$$

In the following, we will follow the procedures proposed by Hassard et al. [18] to figure out the necessary quantities:

$$
\begin{gathered}
g_{20}=\frac{1}{4}\left[\frac{\partial^{2} F_{1}}{\partial y_{1}^{2}}-\frac{\partial^{2} F_{1}}{\partial y_{2}^{2}}+2 \frac{\partial^{2} F_{2}}{\partial y_{1} \partial y_{2}}\right. \\
\left.+i\left(\frac{\partial^{2} F_{2}}{\partial y_{1}^{2}}-\frac{\partial^{2} F_{2}}{\partial y_{2}^{2}}-2 \frac{\partial^{2} F_{1}}{\partial y_{1} \partial y_{2}}\right)\right], \\
g_{11}=\frac{1}{4}\left[\frac{\partial^{2} F_{1}}{\partial y_{1}^{2}}+\frac{\partial^{2} F_{1}}{\partial y_{2}^{2}}+i\left(\frac{\partial^{2} F_{2}}{\partial y_{1}^{2}}+\frac{\partial^{2} F_{2}}{\partial y_{2}^{2}}\right)\right], \\
g_{02}=\frac{1}{4}\left[\frac{\partial^{2} F_{1}}{\partial y_{1}^{2}}-\frac{\partial^{2} F_{1}}{\partial y_{2}^{2}}-2 \frac{\partial^{2} F_{2}}{\partial y_{1} \partial y_{2}}\right. \\
\left.+i\left(\frac{\partial^{2} F_{2}}{\partial y_{1}^{2}}-\frac{\partial^{2} F_{2}}{\partial y_{2}^{2}}+2 \frac{\partial^{2} F_{1}}{\partial y_{1} \partial y_{2}}\right)\right], \\
g_{21}=G_{21}+2 G_{110}^{1} w_{11}^{1}+G_{101}^{1} w_{20}^{1}+2 G_{110}^{2} w_{11}^{2}+G_{101}^{2} w_{20}^{2},
\end{gathered}
$$

where

$$
\begin{aligned}
& G_{21}=\frac{1}{8}[ \frac{\partial^{3} F_{1}}{\partial y_{1}^{3}}+\frac{\partial^{3} F_{1}}{\partial y_{1} \partial y_{2}^{2}}+\frac{\partial^{3} F_{2}}{\partial y_{1}^{2} \partial y_{2}}+\frac{\partial^{3} F_{2}}{\partial y_{2}^{3}} \\
&\left.+i\left(\frac{\partial^{3} F_{2}}{\partial y_{1}^{3}}+\frac{\partial^{3} F_{2}}{\partial y_{1} \partial y_{2}^{2}}-\frac{\partial^{3} F_{1}}{\partial y_{1}^{2} \partial y_{2}}-\frac{\partial^{3} F_{1}}{\partial y_{2}^{3}}\right)\right], \\
& G_{110}^{1}=\frac{1}{2}\left[\frac{\partial^{2} F_{1}}{\partial y_{1} \partial y_{3}}+\frac{\partial^{2} F_{2}}{\partial y_{2} \partial y_{3}}+i\left(\frac{\partial^{2} F_{2}}{\partial y_{1} \partial y_{3}}-\frac{\partial^{2} F_{1}}{\partial y_{2} \partial y_{3}}\right)\right], \\
& G_{110}^{2}=\frac{1}{2}\left[\frac{\partial^{2} F_{1}}{\partial y_{1} \partial y_{4}}+\frac{\partial^{2} F_{2}}{\partial y_{2} \partial y_{4}}+i\left(\frac{\partial^{2} F_{2}}{\partial y_{1} \partial y_{4}}-\frac{\partial^{2} F_{1}}{\partial y_{2} \partial y_{4}}\right)\right], \\
& G_{101}^{1}=\frac{1}{2}\left[\frac{\partial^{2} F_{1}}{\partial y_{1} \partial y_{3}}-\frac{\partial^{2} F_{2}}{\partial y_{2} \partial y_{3}}+i\left(\frac{\partial^{2} F_{2}}{\partial y_{1} \partial y_{3}}+\frac{\partial^{2} F_{1}}{\partial y_{2} \partial y_{3}}\right)\right], \\
& G_{101}^{2}=\frac{1}{2}\left[\frac{\partial^{2} F_{1}}{\partial y_{1} \partial y_{4}}-\frac{\partial^{2} F_{2}}{\partial y_{2} \partial y_{4}}+i\left(\frac{\partial^{2} F_{2}}{\partial y_{1} \partial y_{4}}+\frac{\partial^{2} F_{1}}{\partial y_{2} \partial y_{4}}\right)\right], \\
& w_{11}^{1}=-\frac{1}{4 \lambda_{3}\left(c_{0}\right)}\left(\frac{\partial^{2} F_{3}}{\partial y_{1}^{2}}+\frac{\partial^{2} F_{3}}{\partial y_{2}^{2}}\right),
\end{aligned}
$$




$$
\begin{gathered}
w_{11}^{2}=-\frac{1}{4 \lambda_{4}\left(c_{0}\right)}\left(\frac{\partial^{2} F_{4}}{\partial y_{1}^{2}}+\frac{\partial^{2} F_{4}}{\partial y_{2}^{2}}\right), \\
w_{20}^{1}=\frac{1}{4\left(2 i \omega_{0}-\lambda_{3}\left(c_{0}\right)\right)}\left(\frac{\partial^{2} F_{3}}{\partial y_{1}^{2}}-\frac{\partial^{2} F_{3}}{\partial y_{2}^{2}}-2 i \frac{\partial^{2} F_{3}}{\partial y_{1} \partial y_{2}}\right), \\
w_{20}^{2}=\frac{1}{4\left(2 i \omega_{0}-\lambda_{3}\left(c_{0}\right)\right)}\left(\frac{\partial^{2} F_{4}}{\partial y_{1}^{2}}-\frac{\partial^{2} F_{4}}{\partial y_{2}^{2}}-2 i \frac{\partial^{2} F_{4}}{\partial y_{1} \partial y_{2}}\right), \\
C_{1}(0)=\frac{i}{2 \omega_{0}}\left[g_{20} g_{11}-2\left|g_{11}\right|^{2}-\frac{1}{3}\left|g_{02}\right|^{2}\right]+\frac{1}{2} g_{21} .
\end{gathered}
$$

Through some tedious calculations, one can get

$$
\begin{gathered}
g_{20}=g_{11}=g_{02}=G_{21}=G_{110}^{1}=w_{20}^{1}=0, \\
G_{110}^{2}=-\frac{1}{2}(r l+s m)+\frac{i}{2}(r m-s l), \quad w_{11}^{2}=-\frac{p^{2}}{2 \lambda_{4}}, \\
G_{101}^{2}=-\frac{1}{2}(r l-s m)+\frac{i}{2}(r m+s l), \\
w_{20}^{2}=-\frac{p^{2}}{2\left(2 i \omega_{0}-\lambda_{3}\right)} .
\end{gathered}
$$

Note that in (20) and (22) it is not necessary to calculate $w_{11}^{1}, G_{101}^{1}$, if one wants to obtain expression of $g_{21}$. Its expression is calculated as follows:

$$
\begin{aligned}
g_{21}= & \left(p^{2}(r l+s m)\left(2 \lambda_{3}^{2}+8 \omega_{0}^{2}+2 \lambda_{4} \omega_{0}\right)\right. \\
& \left.+\lambda_{3} \lambda_{4} p^{2}(r l-s m)\right) \\
& \times\left(4 \lambda_{4}\left(\lambda_{3}^{2}+4 \omega_{0}^{2}\right)\right)^{-1} \\
+ & i\left(p^{2}(r l+s m)\left(2 \lambda_{3}^{2}+8 \omega_{0}^{2}\right)\right. \\
& \left.\quad+2 \lambda_{4} \omega_{0} p^{2}(r l-s m)-\lambda_{3} \lambda_{4} p^{2}(s l-r m)\right) \\
\times & \left(4 \lambda_{4}\left(\lambda_{3}^{2}+4 \omega_{0}^{2}\right)\right)^{-1},
\end{aligned}
$$

in which $i^{2}=-1$ and $l, m, n, p, r, s$ are defined to be the same as those in (19).

Based on the previous analysis, one could calculate the following quantities:

$$
\begin{aligned}
C_{1}(0) & \\
= & \frac{1}{2} g_{21} \\
= & \left(p^{2}(r l+s m)\left(2 \lambda_{3}^{2}+8 \omega_{0}^{2}+2 \lambda_{4} \omega_{0}\right)\right. \\
& \left.\quad+\lambda_{3} \lambda_{4} p^{2}(r l-s m)\right) \\
& \times\left(2 \lambda_{4}\left(\lambda_{3}^{2}+4 \omega_{0}^{2}\right)\right)^{-1} \\
+ & i\left(p^{2}(r l+s m)\left(2 \lambda_{3}^{2}+8 \omega_{0}^{2}\right)\right. \\
& \left.\quad+2 \lambda_{4} \omega_{0} p^{2}(r l-s m)-\lambda_{3} \lambda_{4} p^{2}(s l-r m)\right) \\
& \times\left(2 \lambda_{4}\left(\lambda_{3}^{2}+4 \omega_{0}^{2}\right)\right)^{-1},
\end{aligned}
$$

$$
\begin{aligned}
& \mu_{2}=-\operatorname{Re} \frac{C_{1}(0)}{\alpha^{\prime}(0)} \\
& =-\left(p^{2}(r l+s m)\left(2 \lambda_{3}^{2}+8 \omega_{0}^{2}+2 \lambda_{4} \omega_{0}\right)\right. \\
& \left.+\lambda_{3} \lambda_{4} p^{2}(r l-s m)\right) \\
& \times\left(\lambda_{4}\left(\lambda_{3}^{2}+4 \omega_{0}^{2}\right)\right)^{-1} \\
& \times\left(\left[b^{2}(a+1)^{4}+\operatorname{ade}(a+1)^{3}\right.\right. \\
& \left.\left.+a b d e(a+1)^{2}+a b^{2} d e(a+1)+a^{2} d^{2} e^{2}\right]\right) \\
& \times\left(a b^{2}(a+1)^{3}+a^{2} d e(a+1)^{2}\right)^{-1}, \\
& \tau_{2} \\
& =-\frac{\left(\operatorname{Im} C_{1}(0)+\mu_{2} \omega^{\prime}(0)\right)}{\omega_{0}} \\
& =\left(p^{2}(r l+s m)\right. \\
& \left.\times\left[2 \omega_{0}^{2} \lambda_{3}^{2}+2 \lambda_{4} \omega_{0}^{3}+8 \omega_{0}^{4}-(a+1)\left(2 \lambda_{3}^{2}+8 \omega_{0}^{2}\right)\right]\right) \\
& \times\left(2 \lambda_{4} \omega_{0}(a+1)\left(4 \omega_{0}^{2}+\lambda_{3}^{2}\right)\right)^{-1} \\
& -\left(p^{2}(r l-s m)\left[\lambda_{3} \lambda_{4} \omega_{0}^{2}-2 \lambda_{4} \omega_{0}(a+1)\right]\right. \\
& \left.+\lambda_{3} \lambda_{4} p^{2}(a+1)(s l-r m)\right) \\
& \times\left(2 \lambda_{4} \omega_{0}(a+1)\left(4 \omega_{0}^{2}+\lambda_{3}^{2}\right)\right)^{-1}, \\
& \beta_{2} \\
& \begin{aligned}
= & 2 \operatorname{Re} C_{1}(0) \\
= & \left(p^{2}(r l+s m)\left(2 \lambda_{3}^{2}+8 \omega_{0}^{2}+2 \lambda_{4} \omega_{0}\right)\right. \\
& \left.+\lambda_{3} \lambda_{4} p^{2}(r l-s m)\right) \\
& \times\left(4 \lambda_{4}\left(\lambda_{3}^{2}+4 \omega_{0}^{2}\right)\right)^{-1},
\end{aligned}
\end{aligned}
$$

where $\alpha^{\prime}(0)=\operatorname{Re}\left(\lambda^{\prime}\left(c_{0}\right)\right), \omega^{\prime}(0)=\operatorname{Im}\left(\lambda^{\prime}\left(c_{0}\right)\right)$, and $\lambda_{3}, \lambda_{4}$, are those given in Section 5.2. Now one arrives at the following result.

Theorem 3. System (2) exhibits a Hopf bifurcation at the equilibrium $O(0,0,0,0)$ as $c$ passes through $c_{0}$, with the properties below:

(a) if $\mu_{2}>0(<0)$, the Hopf bifurcation is supercritical (subcritical) and bifurcating periodic solutions exist for $c>c_{0}\left(<c_{-} 0\right)$;

(b) if $\beta_{2}<0(>0)$, the bifurcating periodic solutions are orbitally stable (unstable);

(c) if $\tau_{2}>0(<0)$, the period of bifurcating periodic solutions increases (decreases).

5.3. Numerical Simulations. An example of system (2) is given with $a=10, b=8 / 3, d=2$, and $e=12$. According to Theorem 2, one has $c_{0}=1.2182$. And it follows from the results in Section 5.2 and some tedious calculations that

$$
\mu_{2}=0.9249, \quad \beta_{2}=-0.2033, \quad \tau_{2}=-0.0429 .
$$




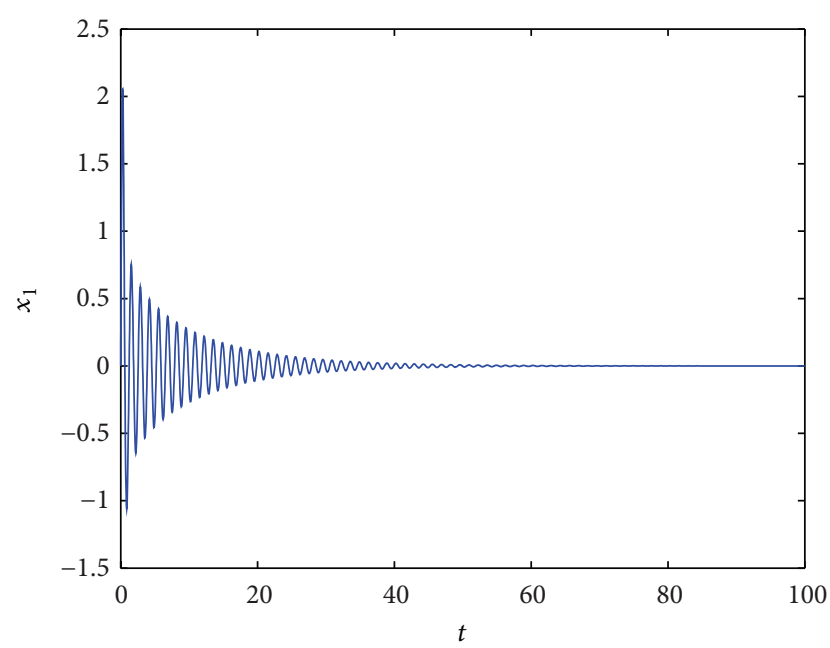

FIGURE 13: Waveform for system (2) with $c=1$.

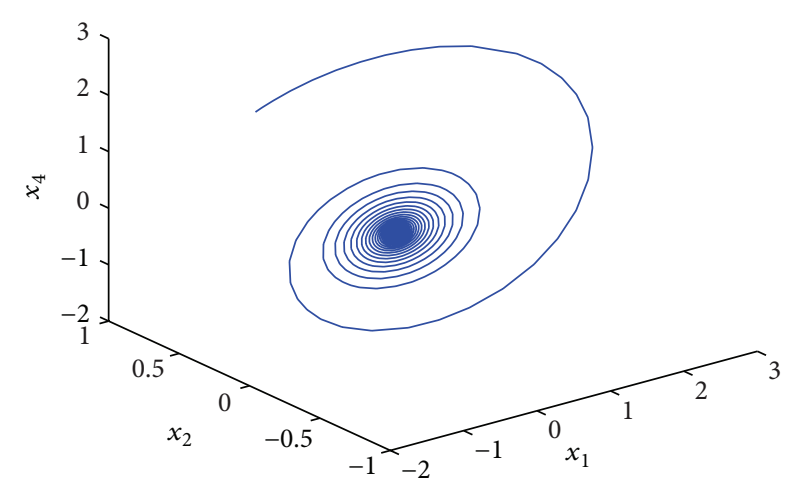

FIGURE 14: Phase portrait of (2) with $c=1$ in $\left(x_{1}, x_{2}, x_{4}\right)$ space.

In the light of Theorem 3, since $\mu_{2}>0$, the Hopf bifurcation is supercritical, which means that the equilibrium $O(0,0,0,0)$ of system (2) is stable when $c<c_{0}$, as shown in Figures 13 and 14. The equilibrium losses its stability and a Hopf bifurcation occurs when $c$ increases past $c_{0}$. That is, a family of periodic solutions bifurcate from the equilibrium point and each individual periodic solution is stable for $\beta_{2}<0$, as shown in Figures 15 and 16. Since $\tau_{2}<0$, the period of bifurcating periodic solutions decreases with $c$ increasing.

\section{Conclusions}

In this paper, a new $4 \mathrm{D}$ hyperchaotic system with only one equilibrium point is presented based on the Lorenz system. Some basic dynamical properties, such as the Lyapunov exponents, bifurcation diagram, fractal dimensions, and hyperchaotic behaviors are investigated. Furthermore, the compound structure and forming mechanism of the new hyperchaotic attractor are revealed via a controlled system with constant controllers. Consequently, it is shown that the new hyperchaotic attractor has a compound structure which can be obtained by merging together two single scroll attractors after performing one mirror operation. Moreover, it is also found that the two single scroll attractors merely

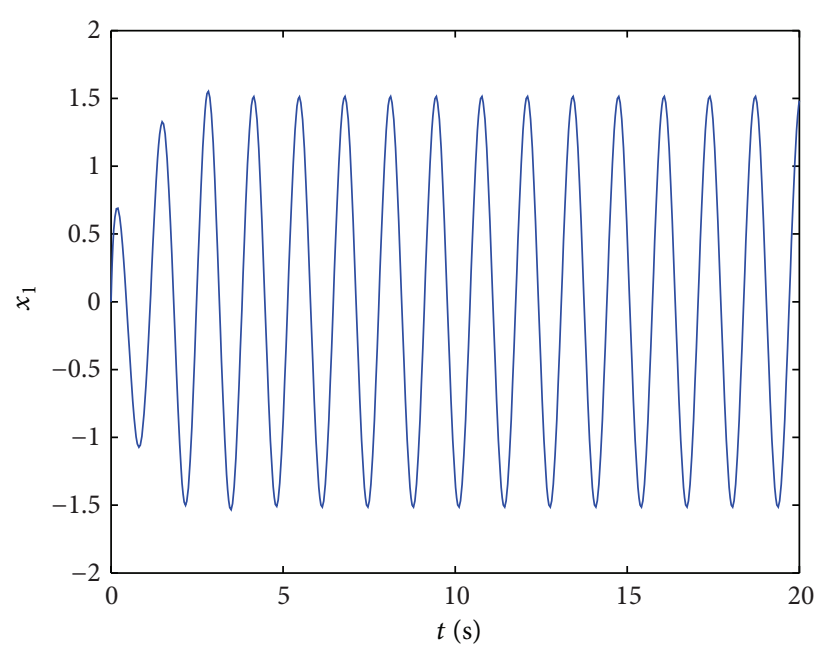

Figure 15: Waveform of system (2) with $c=2.5$.

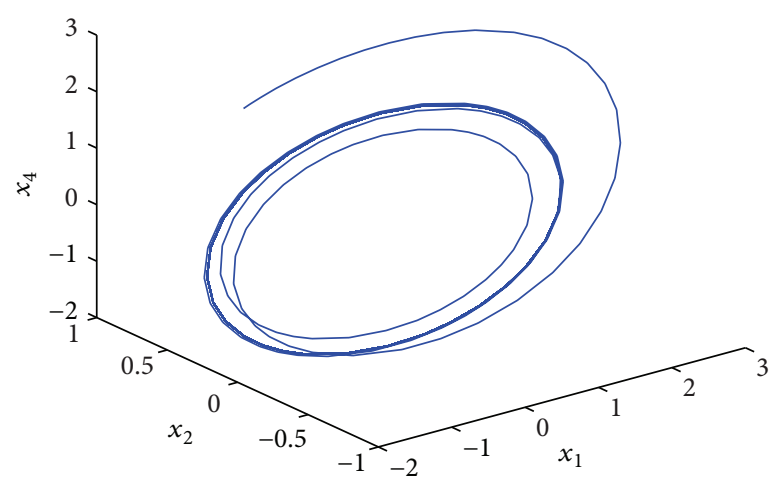

FIGURE 16: Phase diagram for (2) with $c=2.5$ in $\left(x_{1}, x_{2}, x_{4}\right)$ space.

originate from some simple limit circles. In addition, the Hopf bifurcation occurs in this system when the bifurcation parameter exceeds a critical value. The direction of the Hopf bifurcation and the stability of bifurcating periodic solutions are analyzed in detail. Finally, some numerical simulations are also carried out to illustrate the results. There are still some interesting dynamical behaviors about this system, which deserve to be further investigated. It is believed that the system will have some useful applications in various chaosbased systems.

\section{Acknowledgments}

This work is supported by the Specialized Research Fund for the Doctoral Program of Higher Education of China (no. 20093401120001), the Natural Science Foundation of Anhui Province (no. 11040606 M12), the Natural Science Foundation of Anhui Education Bureau (no. KJ2010A035), and the 211 Project of Anhui University (no. KJJQ1102).

\section{References}

[1] E. N. Lorenz, "Deterministic non-periodic flows," Journal of the Atmospheric Sciences, vol. 20, pp. 130-141, 1963. 
[2] G. Chen and T. Ueta, "Yet another chaotic attractor," International Journal of Bifurcation and Chaos in Applied Sciences and Engineering, vol. 9, no. 7, pp. 1465-1466, 1999.

[3] J. Lü and G. Chen, "Dynamical analysis of a new chaotic attractor," International Journal of Bifurcation and Chaos in Applied Sciences and Engineering, vol. 12, no. 5, pp. 1001-1015, 2002.

[4] C. Liu, T. Liu, L. Liu, and K. Liu, "A new chaotic attractor," Chaos, Solitons and Fractals, vol. 22, no. 5, pp. 1031-1038, 2004.

[5] X. Zhou, Y. Wu, Y. Li, and Z. Wei, "Hopf bifurcation analysis of the Liu system," Chaos, Solitons and Fractals, vol. 36, no. 5, pp. 1385-1391, 2008.

[6] R. A. van Gorder and S. R. Choudhury, "Analytical Hopf bifurcation and stability analysis of T system," Communications in Theoretical Physics, vol. 55, no. 4, pp. 609-616, 2011.

[7] O. E. Rössler, "An equation for hyperchaos," Physics Letters A, vol. 71, no. 2-3, pp. 155-157, 1979.

[8] S. Banerjee, A. Das, D. Ghosh, M. Mitra, and A. R. Chowdhury, "Existence of hyperchaos and its control in KuramotoShivashinky equation," International Journal of Nonlinear Science, vol. 11, no. 3, pp. 338-347, 2011.

[9] Z. Chen, Y. Yang, G. Qi, and Z. Yuan, "A novel hyperchaos system only with one equilibrium," Physics Letters A, vol. 360, no. 6, pp. 696-701, 2007.

[10] B. Nana and P. Woafo, "Synchronized states in a ring of four mutually coupled oscillators and experimental application to secure communications," Communications in Nonlinear Science and Numerical Simulation, vol. 16, no. 4, pp. 1725-1733, 2011.

[11] D. Cafagna and G. Grassi, "New 3D-scroll attractors in hyperchaotic Chua's circuits forming a ring," International Journal of Bifurcation and Chaos in Applied Sciences and Engineering, vol. 13, no. 10, pp. 2889-2903, 2003.

[12] K. Thamilmaran, M. Lakshmanan, and A. Venkatesan, "Hyperchaos in a modified canonical Chua's circuit," International Journal of Bifurcation and Chaos in Applied Sciences and Engineering, vol. 14, no. 1, pp. 221-243, 2004.

[13] Y. Li, W. K. S. Tang, and G. Chen, "Hyperchaos evolved from the generalized Lorenz equation," International Journal of Circuit Theory and Applications, vol. 33, no. 4, pp. 235-251, 2005.

[14] S. Pang and Y. Liu, "A new hyperchaotic system from the Lü system and its control," Journal of Computational and Applied Mathematics, vol. 235, no. 8, pp. 2775-2789, 2011.

[15] C. Sparrow, The Lorenz Equations: Bifurcations, Chaos, and Strange Attractors, Springer, New York, NY, USA, 1982.

[16] J. Guckenheimer and P. Holmes, Nonlinear Oscillations, Dynamical Systems and Bifurcation of Vector Field, Springer, New York, NY, USA, 1983.

[17] Y. A. Kuznetsov, Elements of Applied Bifurcation Theory, Springer, New York, NY, USA, 1998.

[18] B. D. Hassard, N. D. Kazarinoff, and Y. H. Wan, Theory and Application of Hopf Bifurcation, Cambridge University Press, Cambridge, UK, 1982. 


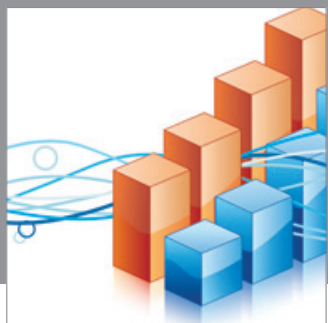

Advances in

Operations Research

mansans

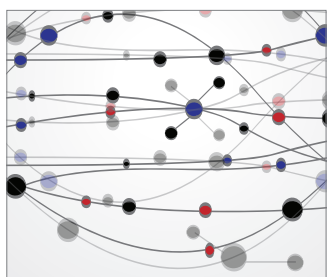

The Scientific World Journal
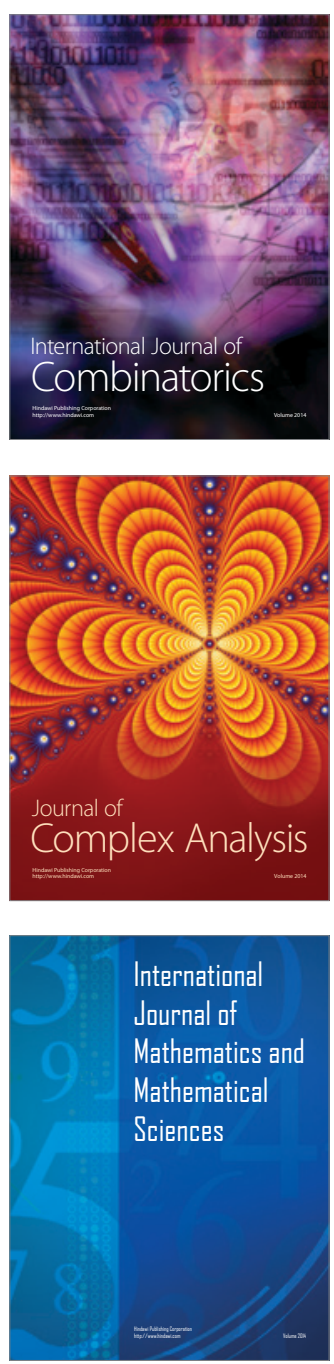
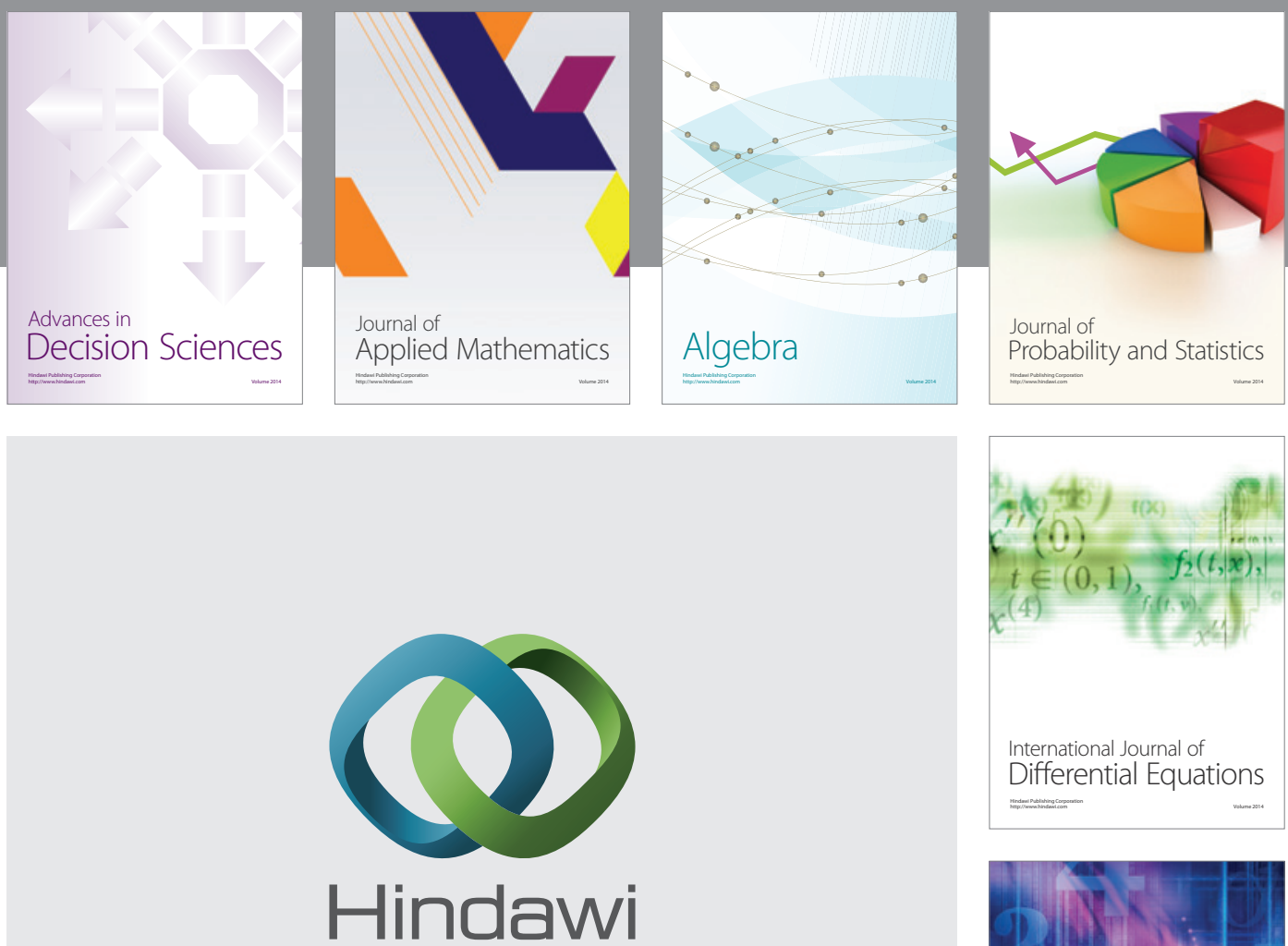

Submit your manuscripts at http://www.hindawi.com
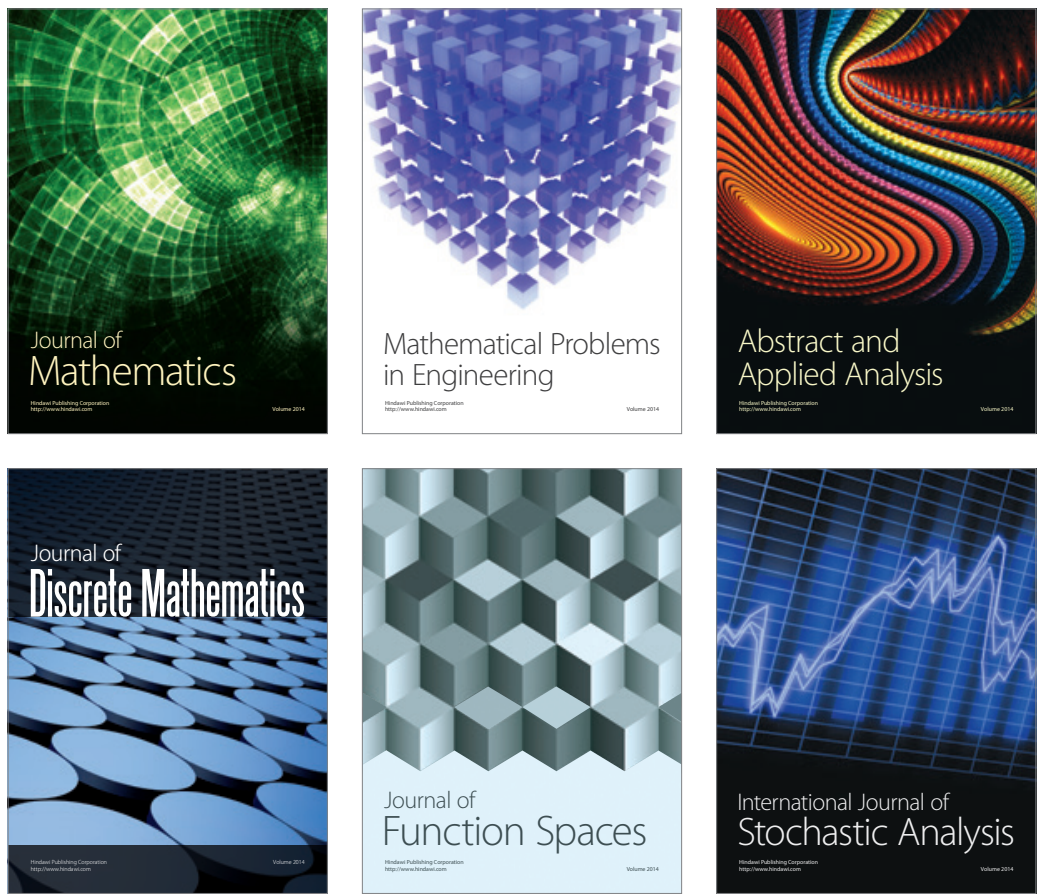

Journal of

Function Spaces

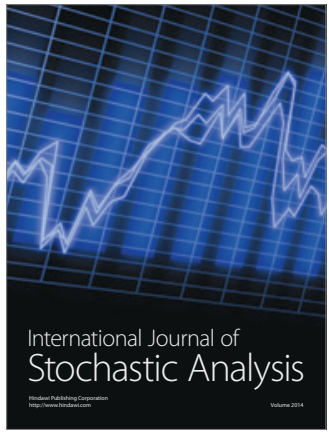

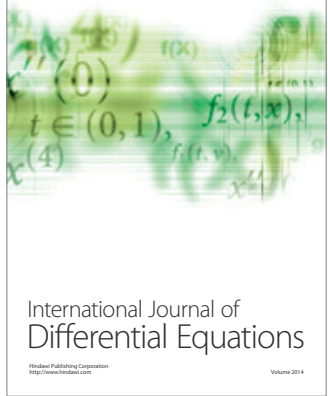
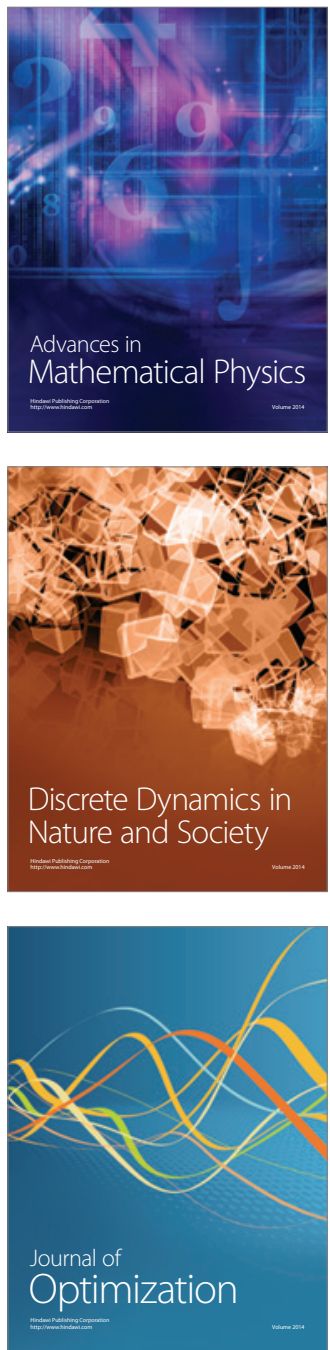